UCM-37-1994

\title{
Green function approach to interface states in band-inverted junctions
}

\author{
Francisco Domínguez-Adame \\ Departamento de Física de Materiales, Facultad de Físicas, Universidad Complutense, E-28040 \\ Madrid, Spain
}

Typeset using REVTEX 
Narrow-gap semiconductor compounds like $\mathrm{Pb}_{1-x} \mathrm{Sn}_{x} \mathrm{Te}$ and $\mathrm{Hg}_{1-x} \mathrm{Cd}_{x} \mathrm{Te}$ present band inversion under compositional variation. In a band-inverted heterojunction the fundamental gap, defined as the difference between $\Gamma_{6}$ and $\Gamma_{8}$ energies, has opposite signs on each side [1]. Type III superlattices with band inversion of $\mathrm{CdTe} / \mathrm{HgTe}$ and $\mathrm{PbTe} / \mathrm{SnTe}$ has been successfully grown in the past [2, [3]. One of the most conspicuous characteristic of bandinverted heterojunctions is the existence of interface states lying within the fundamental gap, provided that the two gaps overlap 4 [ 8 . In IV-VI compounds those interface states are properly decribed by means of a two-band model using the effective $\mathbf{k} \cdot \mathbf{p}$ approximation. On the contrary, the analysis is more complex in II-VI compounds due to mixing with heavy-hole states since non centro-symmetry effects are not negligible in this case. The equation governing conduction- and valence-band envelope-functions in a simple two-band model, neglecting far-band corrections, is a Dirac-like equation. Exact solutions can then be found in view of this analogy because one can use elaborated techniques like those related to supersymmetric quantum mechanics [7]. The aim of this paper is to present an alternative way of solution based on the so called point interaction potentials [9:10] (any arbitrary sharply peaked potential approaching the $\delta$-function limit) along with a Green function method. We believe that our treatment gives a very intuitive explanation of the origin of interfaces states while other approaches may obscure the way how those states arise. Moreover, the effects of external electric and magnetic fields can be included in a straightforward fashion, as we will show later.

In the effective-mass approximation the electronic wave function is a sum of products of Bloch functions at the band-edge with slowly varying envelope-functions. The two-band model Hamiltonian in the absence of external fields is of the form

$$
\mathcal{H}=v_{\perp} \alpha_{y} p_{y}+v_{z} \alpha_{z} p_{z}+\frac{1}{2} \beta E_{G}(z),
$$

where the $Z$ axis is perpendicular to the heterojunction, $E_{G}(z)$ stands for the position dependent gap, $\alpha_{y}, \alpha_{z}$ and $\beta$ are the usual $4 \times 4$ Dirac matrices, $v_{\perp}$ and $v_{z}$ are interband matrix elements having dimensions of velocity. As usual, it is assumed that these matrix 
elements are constant through the whole heterostructure due to the similarity of the zone centre in both semiconductors. Since the gap depends only upon $z$, the transversal momentum is a constant of motion and we can set the $Y$ axis parallel to this component. In the two-band case there are four envelope-functions including spin and we arrange them in a four component vector $F(\mathbf{r})$. This vector satisfies the equation

$$
\mathcal{H} F(\mathbf{r})=[E-V(z)] F(\mathbf{r})
$$

where $V(z)$ gives the position of the gap centre. It is understood that the growth direction is [111]. The way $V(z)$ changes from one layer to another is not well understood but, assuming that the interface states spread over distances much larger than the interface region, we can confidently consider it as a step-like function. Accordingly we take

$$
\begin{gathered}
E_{G}(z)=E_{G L} \theta(-z)+E_{G R} \theta(z), \\
V(z)=V_{L} \theta(-z)+V_{R} \theta(z),
\end{gathered}
$$

$\theta$ being the Heaviside step function. Here, the subscripts $L$ and $R$ mean left and right sides of the heterojunction, respectively.

As we have already mentioned above, the momentum perpendicular to the interface is conserved, and therefore we look for solutions of the form

$$
F(\mathbf{r})=F(z) \exp \left(\frac{i}{\hbar} \mathbf{r}_{\perp} \cdot \mathbf{p}_{\perp}\right)
$$

to Eq. (2). The function $F(z)$ satisfies the following equation

$$
\left(\alpha_{y} v_{\perp} p_{\perp}+\alpha_{z} v_{z} p_{z}+\frac{1}{2} \beta E_{G}(z)-E+V(z)\right) F(z)=0 .
$$

A simple way to solve this equation is the Feynman-Gell-Mann ansatz [11

$$
F(z)=\left(\alpha_{y} v_{\perp} p_{\perp}+\alpha_{z} v_{z} p_{z}+\frac{1}{2} \beta E_{G}(z)+E-V(z)\right) \chi(z) .
$$

After a little algebra we obtain

$$
\left\{-\frac{d^{2}}{d z^{2}}+\frac{1}{\hbar^{2} v_{z}^{2}}\left[\frac{1}{4} E_{G}(z)^{2}-[E-V(z)]^{2}+v_{\perp}^{2} p_{\perp}^{2}\right]-i \Delta \alpha_{z}(\beta-\lambda) \delta(z)\right\} \chi(z)=0 .
$$


For brevity we have defined

$$
\begin{aligned}
\Delta & =\frac{E_{G R}-E_{G L}}{2 \hbar v_{z}}, \\
\lambda & =2 \frac{V_{R}-V_{L}}{E_{G R}-E_{G L}},
\end{aligned}
$$

and we have used the relationships $d \theta( \pm z) / d z= \pm \delta(z)$. Note that in the case of bandinverted heterojunction $E_{G R} E_{G L}<0$.

It is worth mentioning that Eq. (7) is nothing but a Klein-Gordon equation with scalarlike and electrostatic-like terms depending on position (like a relativistic spinless particle with a position-dependent mass in an electric field as occurs in QED) plus a point interaction potential arising from the discontinuity of the gap and the gap centre. The occurrence of this short-range potential makes it possible the existence of bound states deep in the gap. In order to find the bound states we use a Green function formalism, similar to previously used in the case of the Dirac equation with point interaction potentials [12]. To this end, let us consider the Green function associated to Eq. (7) without the point interaction potential

$$
\left\{-\frac{\partial^{2}}{\partial z^{2}}+\frac{1}{\hbar^{2} v_{z}^{2}}\left[\frac{1}{4} E_{G}(z)^{2}-[E-V(z)]^{2}+v_{\perp}^{2} p_{\perp}^{2}\right]\right\} G\left(z, z^{\prime} ; E\right)=I_{4} \delta\left(z-z^{\prime}\right),
$$

where $I_{4}$ stands for the $4 \times 4$ unity matrix. Since we are interested in bound state levels, boundary conditions reads

$$
\lim _{|z|,\left|z^{\prime}\right| \rightarrow \infty} G(z, z ; E)=0 .
$$

The Green function is a $4 \times 4$ matrix which permits the factorization $G\left(z, z^{\prime} ; E\right)=$ $g\left(z, z^{\prime} ; E\right) I_{4}$, where $g\left(z, z^{\prime} ; E\right)$ is a scalar function since the operator in the left hand side of Eq. (9) is scalar.

The solution of Eq. (7) is then simply written out as follows

$$
\begin{aligned}
\chi(z) & =i \Delta \alpha_{z}(\beta-\lambda) \int_{-\infty}^{\infty} d z^{\prime} G\left(z, z^{\prime} ; E\right) \delta\left(z^{\prime}\right) \chi\left(z^{\prime}\right), \\
& =i \Delta \alpha_{z}(\beta-\lambda) g(z, 0 ; E) \chi(0) .
\end{aligned}
$$

It is assumed that the envelope-functions are continuous at the heterojunction so that the value $\chi(0)$ is defined without ambiguities; this is completely different to what found in the 
Dirac equation for point intereaction potentials (see Ref. [9] and references therein). Once the Green function is known, the 4-vector $\chi(z)$ can be obtain and using Eqs. (4) and (6) the envelope-functions are finally determined. Bound state levels can be computed taking the limit $z \rightarrow 0$ in Eq. (11). To obtain nontrivial solutions we require the $4 \times 4$ determinant to vanish. Thus, using the definitions of $\Delta$ and $\lambda$ given in (8) we obtain

$$
\frac{1}{\hbar^{2} v_{z}^{2}}\left[\frac{1}{4}\left(E_{G R}-E_{G L}\right)^{2}-\left(V_{R}-V_{L}\right)^{2}\right]=\left(\frac{1}{g(0,0 ; E)}\right)^{2} .
$$

At this point we would like to stress that we just require the value of the Green function at the origin of the $\left(X, X^{\prime}\right)$ plane if only the bound state levels are needed. In the absence of external fields, as we are considering here, this value is actually not difficult to obtain. Let $u_{+}$and $u_{-}$be two independent, scalar solutions of the Sturm-Liouville problem (7) (dropping the point interaction term), vanishing at $+\infty$ and $-\infty$, respectively. Therefore we can write

$$
g(0,0 ; E)=\frac{u_{+}(0) u_{-}(0)}{W\left[u_{+}, u_{-}\right]}
$$

where $W\left[u_{+}, u_{-}\right]$is the Wronskian of the two solutions. Defining two real parameters

$$
\begin{aligned}
& K_{L}=\frac{1}{\hbar v_{z}} \sqrt{\frac{1}{4} E_{G L}^{2}-\left(E-V_{L}\right)^{2}+v_{\perp}^{2} p_{\perp}^{2}}, \\
& K_{R}=\frac{1}{\hbar v_{z}} \sqrt{\frac{1}{4} E_{G R}^{2}-\left(E-V_{R}\right)^{2}+v_{\perp}^{2} p_{\perp}^{2}},
\end{aligned}
$$

the two independent solutions are $u_{+}=\exp \left(-K_{R} z\right)$ and $u_{-}=\exp \left(K_{L} z\right)$ so that $g(0,0 ; E)=$ $2 /\left(K_{R}+K_{L}\right)$. Using Eq. (12) one finally obtains

$$
K_{R}+K_{L}=\frac{1}{\hbar v_{z}} \sqrt{\frac{1}{4}\left(E_{G R}-E_{G L}\right)^{2}-\left(V_{R}-V_{L}\right)^{2}} .
$$

$K_{R}$ and $K_{L}$ should be real for obtaining exponentially decreasing envelope-functions as $|z| \rightarrow \infty$ and then the gaps must overlap, i. e. $\left(E_{G R}-E_{G L}\right)^{2} / 4>\left(V_{R}-V_{L}\right)^{2}$. This solution agrees with that previously proposed by Korenman and Drew [0]. The reader is referred to Ref. [5] for a fully discussion of its physical implications. Here we stress the main advantages of using our method. First of all, we have restricted ourselves to the case of no external 
potentials. Nevertheless, it is clear that applied electric or magnetic fields can be easily handled with minor modifications of the equations. Note that the crucial point is that one assumes that the Klein-Gordon equation without the point interaction potential arising from the abrupt interface can be solved exactly and the corresponding Green functions is explicitly written out. This is so for a large variety of electric and magnetic field configuration, as pointed out in Ref. [13]. Thus, for instance, it is possible to investigate Landau levels in band-inverted heterojunctions in a rather simple way, instead of using more elaborated mathematical treatments, as those recently carried out by Aggasi [14]. In addition, it is also possible to study confined Stark effect, a topic which remains open in the literature. The second aspect we remark is the fact that there is no need to use an abrupt heterojunction

model, simulated by a step potential. The only requirement is that $K_{R}^{-1}$ and $K_{L}^{-1}$ must be much larger than the interface itself, an implicit assumption when using the envelopefunction formalism. Qualitatively the profile of the heterojunction is soliton-like [7] and, as a consequence, it derivative is a sharply peaked function. Thus the integral equation (11) can be solved by a limiting process, in analogous way to the Dirac equation for sharply peaked functions approaching the $\delta$-function limit [12]. To conclude, we feel that the approach we developed holds in a large variety of cases of practical interest and it may help in a better understanding of interface states in band-inverted heterojunctions.

\section{ACKNOWLEDGMENTS}

This work is supported by UCM through project PR161/93-4811. 


\section{REFERENCES}

[1] K. Seeger, Semiconductor Physcis, (Springer Verlag, Berlin, 1991).

[2] J. P. Faurie, IEEE J. Quantum Electron. QE-22, 1656 (1986).

[3] A. Ishida, M. Aoki, and H. Fujiyasu, J. Appl. Phys. 58, 1901 (1985).

[4] B. A. Volkov and O. A. Pankratov, JETP Lett. 42, 178 (1985).

[5] V. Korenman and H. D. Drew, Phys. Rev. B 35, 6446 (1987).

[6] D. Aggasi and V. Korenman, Phys. Rev. B 37, 10095 (1988).

[7] O. A. Pankratov, Semicond. Sci. Technol. 5, S204 (1990).

[8] V. I. Litvinov and M. Oszwaldowski, Semicond. Sci. Technol. 5, S364 (1990).

[9] F. Domínguez-Adame and E. Maciá, J. Phys. A: Math. Gen. 22, L419 (1989).

[10] E. Maciá and F. Domínguez-Adame, J. Phys. A: Math. Gen. 24, 59 (1991).

[11] R. P. Feynman and M. Gell-Mann, Phys. Rev. 109, 193 (1958).

[12] B. Mendéz and F. Domínguez-Adame, J. Phys. A: Math. Gen. 25, 2065 (1992).

[13] B. Méndez and F. Dom!nguez-Adame, Il Nuovo Cimento B 107, 489 (1992).

[14] D. Agassi, Phys. Rev. B 49, 10393 (1994). 